\title{
Trust Based Routing in Mobile Ad-Hoc Networks
}

\author{
Vinesh H. Patel, Mukesh A. Zaveri, and Hemant Kumar Rath
}

\begin{abstract}
The rapid growth of mobile computing technology and wireless communication have significantly increased the mobile users worldwide. This fact is the reason for the new applications of mobile ad-hoc network and providing reliable communication services to the users. Due to resource scarcity and dynamic network topology, providing reliable service is difficult. The objective of this work is to explore ad-hoc routing issues in MANET. Routing protocols are vulnerable to routing attacks like packet dropping and delayed packet forwarding. Our proposed scheme considers malicious behavior of nodes which causes significant degradation in network performance. To improve the network performance, our scheme includes trust model based on different statistical parameters with their weightage. Our proposed scheme collects information about node and network state parameters related to network layer. Our scheme is implemented in QualNet simulator with modification in the AODV routing protocol by incorporating the trust model into it. The proposed trust model makes sure to uniformly utilize the network resources to increase network lifetime. Simlulation results shows that our proposed trust model based approach increases network lifetime and improves network performance in presence of malicious activities.
\end{abstract}

Index Terms-Mobile ad-hoc network, trusted routing, network lifetime.

\section{INTRODUCTION}

Mobile Ad-hoc NETwork (MANET) is a collection of mobile devices communicating with each other without help of any centralized administration. The devices in MANET can move freely with seamless connectivity and form a self-organized network. MANET doesn't need any existing communication infrastructure. MANET is useful in military communication and other specialized fields such as disaster management and recovery [1], emergency services, and environment monitoring, etc. Military applications cannot rely on fixed infrastructure based communication services in battlefield but MANET is used to quickly self-configure the network and communicate with each other. In emergency services, MANET is used for search and rescue operations and replacement of fixed infrastructure in case of earthquakes, fire etc.

There are various factors like scarcity in network resource, dynamic nature of applications, unstable links and topology, infrastructure less architecture, mobility of nodes etc., affect the performance of MANET. The communication over a dynamic environment like a mobile wireless network is

Manuscript received June 13, 2014; revised August 15, 2014.

Vinesh H. Patel and Mukesh A. Zaveri are with Computer Engineering Department, Sardar Vallabhbhai National Institute of Technology, Surat, Gujarat, India (e-mail: vineshhpatel@gmail.com, mazaveri@coed.svnit.ac.in).

Hemant Kumar Rath is with CTO Networks Lab, Tata Consultancy Services, Bangalore, Karnataka, India (e-mail: hemant.rath@tcs.com). difficult than communicating with a static channel because the link reliability, delay and packet loss are not determined. Hence, providing QoS in ad-hoc network is significantly difficult at the destination, thus degrading user experience. It results into higher packet delay, jitter and lower throughput that decrease the performance of the network [2].

Mobile ad-hoc network can provide new type of services to the mobile users which involve mobility. The rapid progress of mobile computing becomes a powerful trend in the development of Information Technology (IT) as well as commerce and industry fields. The next generation computing is moving towards the era of mobile cloud computing that integrates the cloud computing into the mobile environment and overcomes obstacles related to the performance (e.g., battery life, storage, and bandwidth) [3]. The nature of mobile cloud computing makes usage of mobile ad-hoc networks the easiest solution for the interconnection of devices.

Mobile ad-hoc network consists of mobile nodes connected via wireless link without any centralized network infrastructure. Furthermore, nodes can move freely in the area which results in the rapid change in wireless network's topology. Hence, the dynamic change in topology causes frequent disconnections which result in large packet loss. When a route breaks, the discovery of a new route will most likely introduce delays, which will affect the performance of the network

In multihop networks, optimal routing is a big challenge. The routing protocol should ensure that each session is provided with a route satisfying its QoS requirements (e.g., bandwidth, delay and jitter). Additionally, the routing protocol should avoid network congestion by load balancing between routes in order to utilize the resources optimally [4].

The devices participating in a MANET are predominantly small devices, with limited processing power, memory and storage capacity. The bandwidth is shared by all devices in the surrounding area in wireless communication. Additionally, an increase in network traffic places additional load on the nodes in the network, which in turn increases energy consumption [5]. Therefore, it is challenging to design a technique that utilizes the energy minimally and uniformly.

When the communication ranges of the nodes are limited, nodes agree to cooperate with their neighbors to forward each other's packets and extend the overall communication range of the network [6]. The scenario could be imagined as: the sensor nodes are deployed in a region of interest to sense the temperature. The sensed value may be forwarded to the base station or a powerful node. That information is used to handle the disastrous situation. Furthermore, malicious node can affect the genuine activities of network. The malicious behavior of node can be prevented by including the security 
mechanism in routing protocol. Furthermore, the most of basic routing protocols are not energy aware so that battery of some node in the network drains out quickly as compared to other nodes in network.

\section{THEORETICAL BACKGROUND AND LITERATURE SURVEY}

In this section, we describe the existing routing protocols along with the attacks and solutions to prevent the attacks followed by the existing trust management based routing protocols.

\section{A. Routing Protocols for MANET}

Unicast routing protocols are used to find the correct and efficient route between nodes to deliver messages reliably. Ad-hoc On-demand Distance Vector (AODV) routing protocol [7] is a reactive protocol which minimizes the broadcast of route requests by creating routes on-demand whenever it is needed. Congestion aware routing mechanism [8] has been proposed by using metrics such as MAC layer utilization, queue length and mobility parameter to increase throughput of the network.

Energy is the important issue that needs to be addressed as the nodes of an ad-hoc network have limited battery power [9]. Several researches proposed many algorithm [10], [11] [12] for handling the limited resource in efficient manner. Energy efficient MANET routing protocol that uses load distribution approach has been presented in [13]. The specific goal of the load distribution approach is to balance the energy usage of all mobile nodes by selecting a route with underutilized nodes rather than the shortest route.

Mobile ad-hoc networks contain different type of sensors that are equipped in mobile devices. In multihop wireless network a mobile node needs to relay the packets to other nodes in order to communicate with the remote destination. Malfunctioned mobile nodes frequently drop packets and break routes due to faulty hardware or software and malicious nodes actively break routes to disrupt data transmission [11], [14]. The multihop packet transmission with the help of routing extends the communication range of the network and improves spectral efficiency.

Routing techniques are used to extend the lifetime of the wireless network. Genetic Algorithm (GA) based and Ant Colony Optimization (ACO) based routing algorithms have been proposed to extend the lifetime of the network [15].

\section{B. Attacks on Routing Protocols and Its Solutions}

Attacks on routing protocol are categorized in two categories: active and passive. Though passive attacks do not disrupt the network, they monitor and attempt to figure out valuable information related to the network. Active attacks disrupt the network and degrade the network performance [16]. Though both active and passive attacks are important, this work mainly focuses on active attacks that affect the network performance and network operations.

The nodes that do not cooperate with network functions and only use the services of the network are called selfish nodes. Selfish nodes intentionally drop all the packets that are not destined for it to save the transmission power [17]. Thus, selfish nodes decrease the network performance. In order to detect the selfish nodes, security based encryption and authentication mechanism is used that consumes more energy, making it infeasible to use in mobile devices [18].

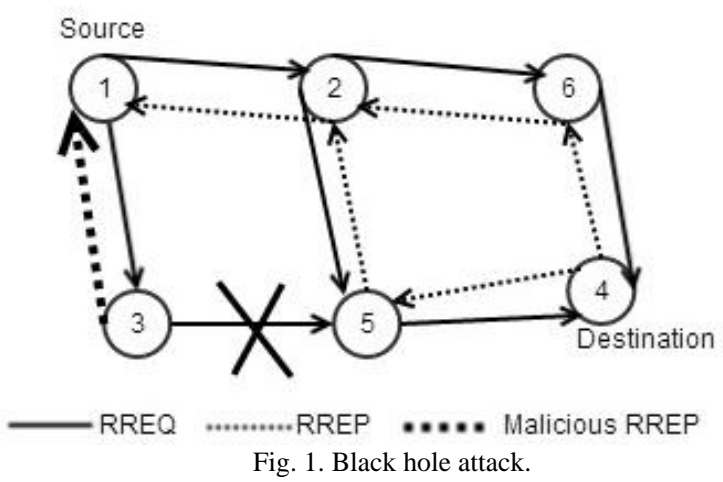

In black hole attack, selfish node advertises the better route through it, and then drops all the data as shown in Fig. 1. Gray hole attack is another form of black hole attack, in which a malicious node drops only selected packets and forwards others, depends on the source or the destination of packets [19]. Hence, black hole and gray hole attacks decrease the packet delivery ratio and network performance. Flooding attack is another malicious behavior performed by malicious nodes. Its goal is to utilize the bandwidth and nodes' resources such as battery and computational power and thereby decrease network lifetime. To prevent these attacks, intrusion detection systems are used to detect the malicious activities and generate alert for intrusions which are less energy consuming as compared to cryptographic solutions [14]. However, intrusion detection systems consume considerable amount of energy in monitoring the network state and finding the malicious signature or pattern to detect the malicious activities.

To counter routing attacks, three techniques have been proposed in [16]. First, prevention techniques are used to secure routing with the authentication and encryption mechanisms based on asymmetric and symmetric cryptography. Second, intrusion detection based technique is used to detect malicious and selfish nodes. It is a set of mechanisms and methods that are used to detect suspect activities and alerts about intrusions. Third, trust management and reputation is based on dynamically evaluating the trust value of each node based on their network activities and performance in the network. Reputation systems use passive monitoring of network activities and recommendation messages between nodes in the network.

Conventional approaches for tackling the security attacks are not feasible for its application to ad-hoc routing because of their high memory requirements and high power consumption. Many trust management frameworks and reputation based frameworks are proposed so far which use different mechanisms to fulfill the security solutions with low memory requirements and low power consumption.

\section{Related Work}

In this section, we describe the existing trust based solutions for routing protocols. Group based Trust Management Scheme (GTMS) [20], [21] for distributed wireless sensor networks have been presented, in which the 
whole group gets a single trust value. GTMS uses a combination of centralized and decentralized trust management approaches that helps in keeping minimum resource utilization at the sensor nodes.

Probabilistic trust management framework [22] has been proposed for pervasive computing, in which trust is computed based on evaluations of previous interactions between devices. The reputation-based trust management scheme in [23] uses trust vote to establish trust among nodes. Value of trust vote is increased with every successful message transmission and decreased with every unsuccessful message transmission from one node to another.

Two trust-based and energy-aware routing protocols, called the Shortest Reliable Route (SRR) and the Best Available Route (BAR) have been presented in [14]. Their goal is to establish stable and reliable routes to reduce the probability of breaking due to the lack of energy, malicious node behavior, and low hardware resources, etc. SRR protocol establishes the shortest route that can satisfy the source node's requirements including energy, trust, and route length. For BAR protocol, the destination node receives multiple routes and selects the most reliable one.

Trust based routing with probability of packet forwarding has been presented in [24]. It measures the direct trust of nodes by the number of packets forwarded and dropped by it. At each node, it increments the trust value on successful forwarding of packets and decrement the trust value upon dropping the packets. This approach is incorporated with Dynamic Source Routing (DSR) protocol and while finding the routing path the trust value is piggybacked with the route request packet. Another trust based routing approach has been presented in [25]. In this approach, each node uses initial trust value as uncertain and its values are updated by opinions of other nodes given time to time to form trust relationship.

Light weight trust based routing has been presented in [26] in which the trust values of neighbor as well as the trust value of routing paths are also maintained. This approach only considers a single parameter, i.e., packet forwarding.

\section{Proposed Trust Model Based Routing}

In this section, we describe the problem statement for routing in MANET and then discuss our proposed trust model to mitigate the problems described.

\section{A. Problem Statement}

In multihop wireless networks, routing protocol is used to transfer packets between nodes via intermediate nodes. Basically routing protocol is divided into three phases: route discovery, packet forwarding and route maintenance. The route discoveries may incur network-wide flooding of routing requests that can consume a considerable amount of the network resources such as energy. Malicious nodes perform malicious activities like packet dropping, flooding, and packet delaying etc., to utilize the limited resources of a node. Breaking the routes increases the packet delivery latency and may cause network partitioning resulting in failure of the multihop communications. Therefore, in order to establish stable routes and maintain continuous traffic flow, it is essential to assess the nodes' competence and reliability in relaying packets to make cognizant routing decisions [14]. The security related solutions to prevent malicious activities includes cryptography and authentication operations which are considered very complex and energy hungry in context of availability of resources in node. Therefore, light weight solution to the problem is required in order to increase throughput of the network.

Furthermore, once the route has been established, the packets are transmitted through that route and the every intermediate node in the route forwards the packet towards destination. The packets are transmitted through same nodes of a path as long as that path is available. Thus decreases the battery level of nodes in the path significantly as compared to other nodes in network. Hence route maintenance phase is required to consider load balancing of packets being forwarded to increase the network lifetime.

\section{B. Proposed Trust Model}

In this section, our proposed trust model is described briefly. The main goal of the model is to provide combined solution for preventing malicious activities and uniform resource utilization by load balancing of packets being forwarded. The trust model represents how to calculate the trust of the routing path by using the trust value of individual nodes. Our trust model creates relationship between trust metrics and network statistics. Our main contribution is to provide a solution for the uniform energy consumption for all the nodes in order to increase network lifetime.

Our trust model mainly consists of two phases: trust formation and trust usage for routing decisions. In trust formation phase, each node collects the network statistics like packets forwarded, packets dropped and packets delayed etc., based on which trust of a node is calculated. Though collection of statistics is performed regularly, it is used only when requested routing path contains the node as intermediate node. Once the route from source to destination is requested, all the intermediate nodes calculate their trust values using equation (1)

$$
T=\sum_{i=0}^{n} a_{i} p_{i}
$$

where $n$ is the number of parameters, $a_{i}$ is weighting factor of $i^{\text {th }}$ parameter and $p_{i}$ is the trust value of $i^{\text {th }}$ parameter.

We then analyze the set of parameters like number of data packets dropped and forwarded, number of control packets dropped and forwarded, remaining energy etc. These parameters are useful not only to prevent malicious activities but also are used to determine the healthiness, congestion, reliability of a link and uniform resource utilization etc.

Legitimate nodes may perform the flooding, initiate route requests, initiate route error etc., activities which may decrease its trust. Therefore, trust model includes weightage of each parameter by considering the effect of the parameter on network performance. Malicious node performs flooding and initiates route request kind of activities repeatedly as compared to legitimate nodes, which keeps trust of legitimate nodes higher than the malicious nodes. 
The following steps are carried out by trust model in order to establish trusted routing path.

1) Initialize trust related network statistics and trust value of all the nodes in network.

2) Update trust related network statistics after all the operations like packet drop, packet forwarding, etc., of each node.

3) When route is to be established, broadcast route request for destination.

4) When the destination receives the route request, it sends route reply along with the trust of path initialized to 0 .

5) All the intermediate nodes receiving route reply calculate their trust value using network statistics and add it to the trust of path in route reply packet.

6) When reply is received by source node first time, it stores the path and starts sending data packets via that path. In case, the source node receives multiple route replies, it compares the trust of newly received path with trust of current path for that destination and stores the path with maximum trust value.

7) The routing path is updated to most trusted path at regular intervals.

\section{Trust Based AODV Routing Protocol}

We incorporate our trust model with AODV routing protocol in order to prevent the malicious behavior and uniform utilization of network resources. The AODV protocol is modified as described below.

1) AODV sends RREP packet for each RREQ packet it receives, thereby enabling AODV to make the destination sends multiple RREP packets for single route request.

2) RREP packet structure is modified to contain trust value of the path.

3) The routing table structure is modified to store the trust value for each entry of source to destination.

4) AODV sends request to update the routing path at regular intervals. Hence, at regular intervals, source node is going to have multiple paths each with its trust value from which one with the maximum trust is selected.

5) The method to handle RREP packet is changed to update the route entry when new path is received with greater trust than current trust value.

6) AODV is modified to send RREQ packet to destination every time thereby disabling the mechanism to initiate RREP packet at intermediate nodes.

\begin{tabular}{|c|c|c|c|c|c|}
\hline $\begin{array}{l}\text { Type } \\
\text { (8bits) }\end{array}$ & $\begin{array}{c}\mathrm{R} \\
\text { (1bit) }\end{array}$ & $\begin{array}{c}\text { A } \\
\text { (1bit) }\end{array}$ & $\begin{array}{c}\text { Reserved }(9 \\
\text { bits })\end{array}$ & $\begin{array}{c}\text { Prefix } \\
\text { Size } \\
\text { (5bits) }\end{array}$ & $\begin{array}{l}\text { Hop } \\
\text { Count } \\
\text { (8bit) }\end{array}$ \\
\hline \multicolumn{6}{|c|}{ Destination IP address (32 bits) } \\
\hline \multicolumn{6}{|c|}{ Destination sequence number (32 bits) } \\
\hline \multicolumn{6}{|c|}{ Original IP address ( 32 bits) } \\
\hline \multicolumn{6}{|c|}{ Lifetime (32 bits) } \\
\hline \multicolumn{6}{|c|}{ Trust of Path (32 bits) } \\
\hline
\end{tabular}

Fig. 2. Modified RREP packet format.

The major difference between original AODV and proposed trust based AODV is that our version of AODV can generate multiple RREP packets and RREP packet is modified to carry trust of the path. Trust based AODV also updates routing path at regular intervals for uniform resource utilization. The modified RREP packet structure is shown in Fig. 2 with the bit information of each field.

\section{Simulation Studies}

In this section, we describe the simulation parameters and its results to justify our trust model. We have used QualNet network simulator [27] to perform trust model enabled and modified AODV simulations. The simulation parameters are shown in Table I. We have implemented the trust model proposed in Section III-C in Qualnet.

TABLE I: SIMULATION PARAMETERS

\begin{tabular}{rll}
\hline \hline No. & Parameter & Value(s) \\
\hline \hline 1. & Simulation time & 5 HOURS \\
2. & Number of nodes & $8,25,50,75,100$ \\
3 & No of malicious nodes & $2,3,5,8,10$ \\
4. & Terrain dimension & $(1500,1500)$ \\
5. & Routing protocol & AODV, Modified AODV \\
6. & Application protocol & CBR \\
7. & Max Speed & 10 m/s \\
8. & Packet size & 1024 bytes \\
9. & Mobility model & Random way point \\
10. & Channel type & Wireless channel \\
11. & Antenna type & Omni directional \\
12. & Transmission range & 350 m \\
13. & MAC protocol & MAC 802.11 \\
14. & Battery model & Residual Life estimator \\
15. & Queue type & FIFO \\
16. & Max queue size & $150 \mathrm{~KB}$ \\
\hline \hline
\end{tabular}

The scenario with two routing paths 1-3-4-5-2 and 1-6-7-8-2 is shown in Fig. 3. The scenario does not contain malicious nodes, so the trust of path mainly depends on the energy of node as compared to other parameters for calculating trust. Therefore, after each trust update interval, alternate paths are selected from source to destination because their trust values changes with time. Fig. 4 shows the graph of trust of paths with respect to time. The graph shows that after each trust update interval, trust of previously selected path is decreased as compared to other path. Therefore, alternate paths are chosen and thereby confirming the load balancing in terms of number of packets forwarded as shown in Fig. 5.

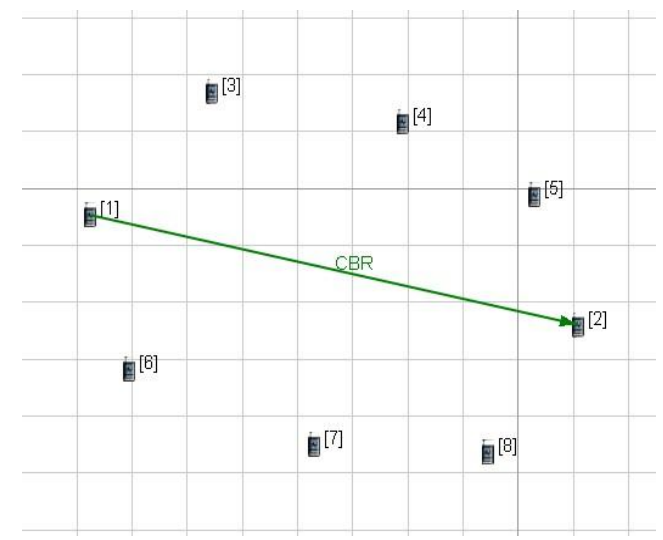

Fig. 3. Scenario with two routing paths. 
TABLE II: RESULTS OF MOBILE SCENARIOS

\begin{tabular}{|c|c|c|c|c|c|c|}
\hline \multirow{2}{*}{ Node density } & \multicolumn{2}{|c|}{ Experiment } & \multirow{2}{*}{$\begin{array}{c}\text { Throughput } \\
\text { (kbps) }\end{array}$} & \multirow{2}{*}{ PDR } & \multirow{2}{*}{$\begin{array}{l}\text { Delay } \\
(\mathrm{MS}) \\
\end{array}$} & \multirow{2}{*}{ Jitter(MS) } \\
\hline & Malicious & Trust & & & & \\
\hline \multirow{4}{*}{25} & $\mathrm{~N}$ & $\mathrm{~N}$ & 6.548 & 79.9 & 27.46 & 16.02 \\
\hline & $\mathrm{N}$ & $\mathrm{Y}$ & 5.562 & 67.9 & 34.90 & 15.51 \\
\hline & $\mathrm{Y}$ & $\mathrm{N}$ & 4.455 & 54.4 & 21.84 & 12.39 \\
\hline & $\mathrm{Y}$ & $\mathrm{Y}$ & 5.192 & 63.4 & 25.16 & 10.12 \\
\hline \multirow{4}{*}{50} & $\mathrm{~N}$ & $\mathrm{~N}$ & 7.514 & 91.7 & 22.95 & 10.90 \\
\hline & $\mathrm{N}$ & $\mathrm{Y}$ & 6.125 & 74.8 & 35.47 & 17.70 \\
\hline & $\mathrm{Y}$ & $\mathrm{N}$ & 5.294 & 64.6 & 21.21 & 10.18 \\
\hline & $\mathrm{Y}$ & $\mathrm{Y}$ & 5.856 & 71.5 & 23.29 & 7.50 \\
\hline \multirow{4}{*}{75} & $\mathrm{~N}$ & $\mathrm{~N}$ & 7.653 & 93.4 & 21.51 & 9.36 \\
\hline & $\mathrm{N}$ & $\mathrm{Y}$ & 6.532 & 79.7 & 8.70 & 17.56 \\
\hline & $\mathrm{Y}$ & $\mathrm{N}$ & 5.314 & 64.8 & 18.71 & 7.72 \\
\hline & $\mathrm{Y}$ & $\mathrm{Y}$ & 7.009 & 85.6 & 26.12 & 8.81 \\
\hline \multirow{4}{*}{100} & $\mathrm{~N}$ & $\mathrm{~N}$ & 7.688 & 93.8 & 21.80 & 9.61 \\
\hline & $\mathrm{N}$ & $\mathrm{Y}$ & 6.861 & 83.7 & 33.64 & 11.88 \\
\hline & $\mathrm{Y}$ & $\mathrm{N}$ & 5.176 & 63.1 & 18.56 & 7.49 \\
\hline & $\mathrm{Y}$ & $\mathrm{Y}$ & 6.633 & 81.0 & 26.66 & 9.11 \\
\hline
\end{tabular}

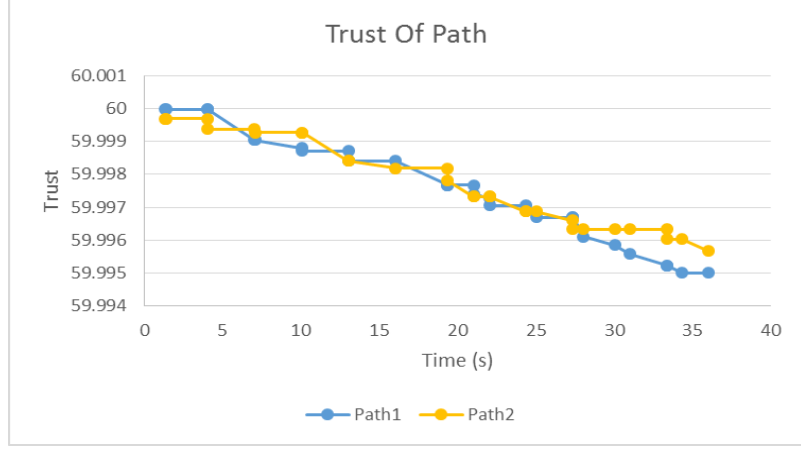

Fig. 4. Trust of path 1 and path 2.

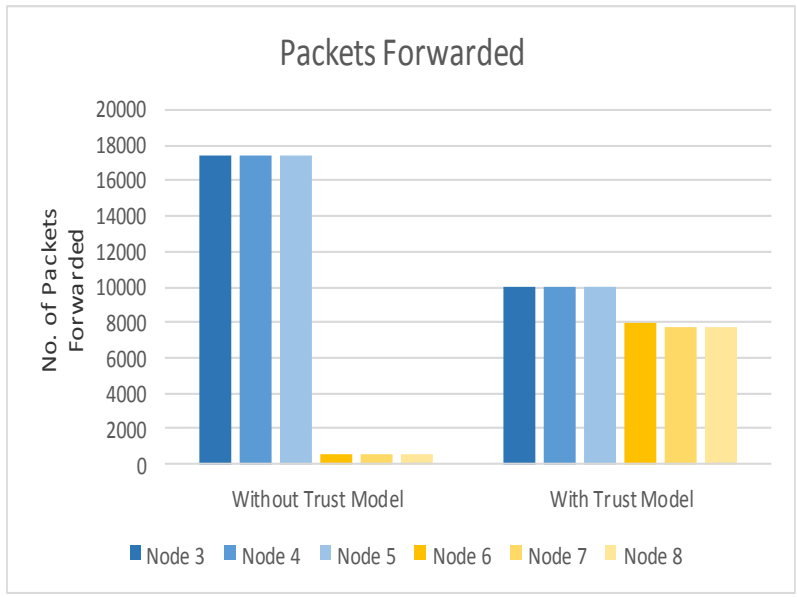

Fig. 5. Packets forwarded by intermediate nodes.

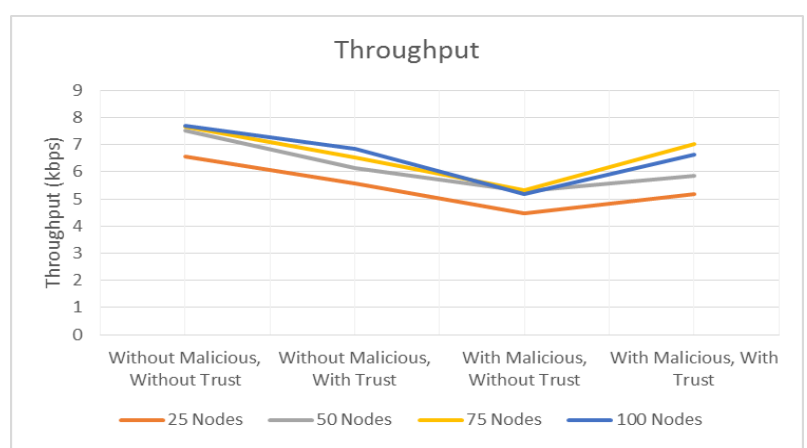

Fig. 6. Throughput of the network.
Along with the specified load balancing scenario, other scenarios are also considered with varying number of nodes as shown in the Table II with all the configurations and the results. Fig. 6 shows the throughput of all the scenarios and all four combinations. The graph shows that in presence of malicious nodes, without using trust model, throughput is decreased and using trust model it is improved.

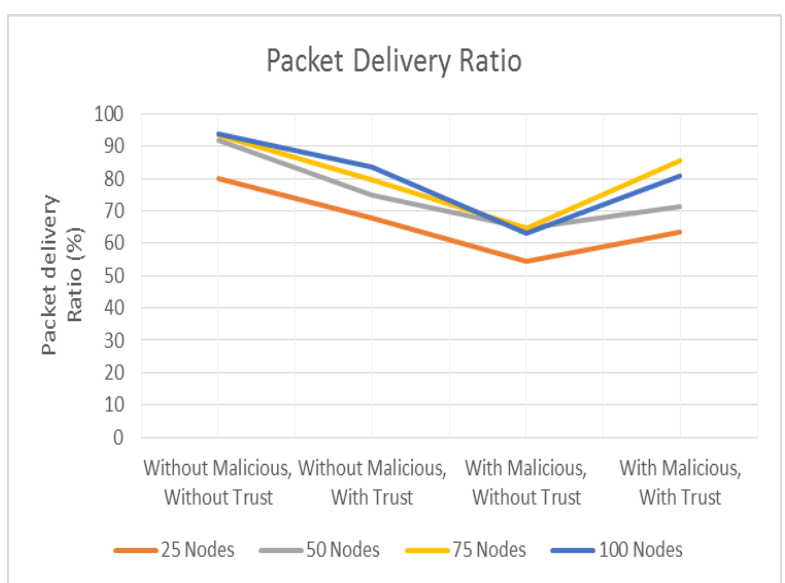

Fig. 7. Packet delivery ratio of the network.

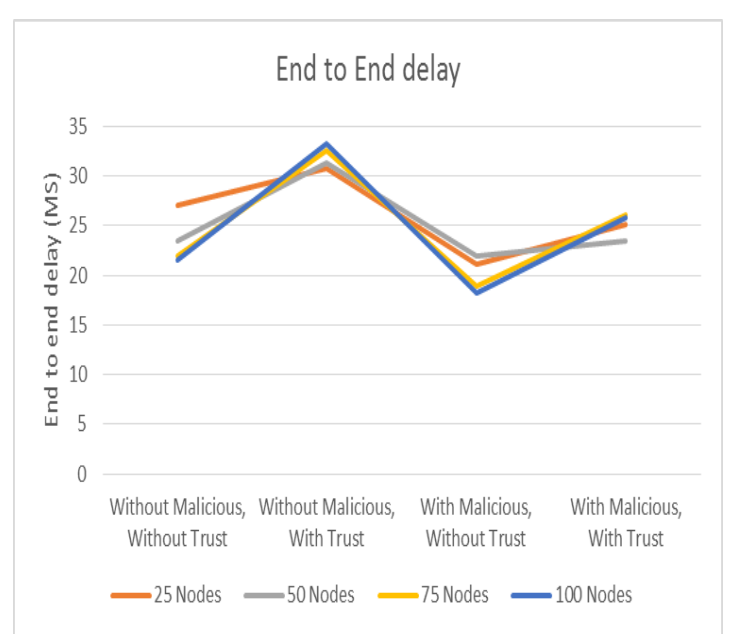

Fig. 8. Avg. end to end delay of network. 
Fig. 7 shows the graph of packet delivery ratio for different number of nodes with all combinations of with and without trust model and malicious activities. The graph shows that packet delivery ratio is improved with node density and in presence of malicious behavior using trust model. End to end delay and jitter are getting increased because of extra routing overhead caused by updating trust of the path at regular intervals as shown in graph of Fig. 8 and Fig. 9 respectively.

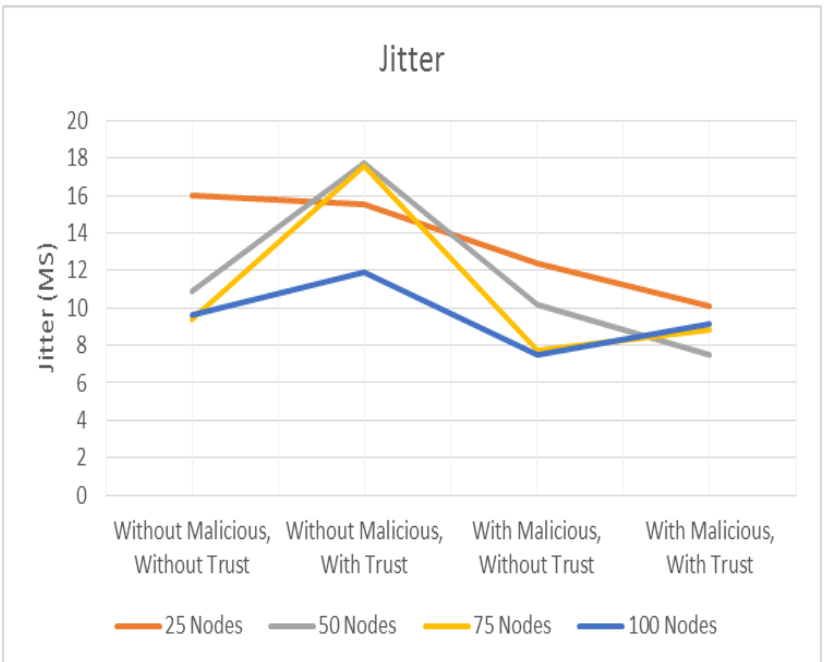

Fig. 9. Avg. Jitter of network.

\section{CONCLUSION AND FUTURE WORK}

In our proposed system, our main aim is to optimize the energy consumption so as to increase the lifetime of the network and to decrease the effect of malicious activities of nodes. With the overview of trust model in routing protocol, we have described various approaches to improve network performance. We have created different to compare the existence and non-existence of trust model.

We have conducted Qualnet based simulations with and without trust model modification of AODV. From the simulation results we have observed that with AODV in place, in presence of malicious nodes in the network, the packet delivery ratio and throughput are degraded. To prevent the malicious behavior of nodes, we have implemented the trust model (modified AODV) to find reliable route resulting in improvement in the packet delivery ratio and throughput of the network. Moreover, even in absence of malicious nodes, routing protocol with trust model also chooses routing path in a way that it utilizes available resources uniformly thereby increases the network lifetime.

We have provided a framework for efficient routing protocol that can be extended to include more parameters to calculate trust of the nodes. By adding parameters from other layers such as Medium Access Control (MAC) and Physical, trust value of the nodes can be recomputed such that the wireless channel condition and other related information can be included in the process. This can further be improved by the use of Machine learning and Genetic algorithm based techniques for dynamic tuning of weightage of all the parameters.

The quality of service parameters like end to end delay and jitter can be more concentrated to improve overall performance of the network. Delay aware routing protocol can be designed to use with trust model as a solution for it. Furthermore, mechanisms like bandwidth estimation can also be integrated with our approach to improve network performance in mobile ad-hoc networks.

\section{REFERENCES}

[1] I. Chlamtac et al., "Mobile ad-hoc networking: Imperatives and challenges," Ad Hoc Networks, vol. 1, no. 1, pp. 13-64, 2003.

[2] V. T. Raisinghani and S. Iyer, "Cross-layer design optimizations in wireless protocol stacks," Computer Communications, vol. 27, no. 4, pp. 213-217, 2006.

[3] M. Satyanarayanan, "Mobile computing: The next decade," in Proc. 1st ACM Workshop on Mobile Cloud Computing and Services: Social Networks and Beyond (MCS), 2010, pp. 5:1-5:6.

[4] S. Khan et al., "Cross-layer optimization for wireless video streaming performance and cost," presented at International Conference on Multimedia and Expo, Amsterdam, July 2005.

[5] P. Kevre and L. Shrivastava, "Compare three reactive routing protocols in grid based cluster wireless sensor network using qualnet simulator," International Journal of Applied Sciences and Engineering Research, vol. 4, no. 4, pp. 532-539, 2014.

[6] A. A. Gohari and V. Rodoplu, "Congestion-aware spatial routing in hybrid high-mobility wireless multihop networks," IEEE Transactions on Mobile Computing, vol. 12, no. 11, pp. 2247-2260, 2013.

[7] C. Perkins, E. Belding-Royer, and S. Das, "Ad-hoc On-Demand Distance Vector (AODV) routing," Mobile Adhoc Networking Working Group Internet draft, vol. 5, no. 2, pp. 32-34, July 2003.

[8] Y. Hu and D. Johnson, "Exploiting Congestion Information in Network and Higher Layer Protocols in Multihop Wireless Ad Hoc Networks," in Proc. the 24th International conference on Distributed computing Systems, vol. 31, no. 8, pp. 301-310, 2004.

[9] A. Goldsmith and S. Wicker, "Design challenges for energy constrained ad-hoc wireless networks," Wireless Communications, vol. 9, no. 4, pp. 8-27, 2002.

[10] N. K. Ray and A. Turuk, "Energy efficient technique for wireless Ad-hoc network," in Proc. International Joint Conference on Information and Communication Technology, 2010, pp. 105-111.

[11] P. Sivasankar, C. Chellappan, and S. Balaji, "Performance of energy efficient routing protocol for MANET," International Journal of Computer Applications, pp. 1-6, 2011.

[12] N. A. Pantazis and D. D. Vergados, "A survey on power control issues in wireless sensor networks," Communications Surveys and Tutorials, vol. 9, no. 4, pp. 86-107, 2007.

[13] D. B. Johnson and S. PalChaudhuri, "Power mode scheduling for Ad Hoc networks," in Proc. International Conference on Network Protocols, 2008, vol. 19, no. 5, pp. 192-193.

[14] M. Mahmaud et al., "Secure and reliable routing protocols for heterogeneous multihop wireless networks," IEEE Transactions on Parallel and Distributed Systems, pp. 1-11, 2013.

[15] K. Rana and M. Zaveri, "Techniques for Efficient Routing in Wireless Sensor network," presented at International Conference on Intelligent Systems and Data Processing, 2011.

[16] A. Abdelaziz, M. Nafaa, and G. Salim, "Survey of routing attacks and countermeasures in mobile Ad Hoc networks," in Proc. 15th International Conference on Computer Modelling and Simulation (UKSim), 2013, pp. 693-698.

[17] E. Y. Vasserman and N. Hopper, "Vampire attacks: Draining life from wireless Ad Hoc sensor networks," IEEE Transaction on Mobile Computing, vol. 12, no. 2, pp. 318-332, 2013.

[18] T. Chen, O. Mehani and R. Boreli, "Trusted routing for VANET," in Proc. International Conference on Intelligent Transport Systems Telecommunications, October 20-22, 2009, pp. 647-652.

[19] B. Yu and B. Xiao, "Detecting selective forwarding attacks in wireless sensor networks," in Proc. International Symposium and Parallel and Distributed Processing, April 25-29, 2006.

[20] R. Shaikh, H. Jameel, S. Lee, and S. Rajput, "Trust management problem in distributed wireless sensor networks," in Proc. International Conference on Embedded and Real-Time Computing Systems and Applications, 2006, pp. 411-414.

[21] R. Shaikh, H. Jameel, and H. Lee, "Group-based trust management scheme for clustered wireless sensor networks," IEEE transactions on Parallel and Distributed Systems, vol. 20, no. 11, pp. 1698-1712, 2009.

[22] M. Deno and T. Sun, "Probabilistic trust management in pervasive computing," in Proc. International Conference on Embedded and Ubiquitous Computing, 17-20 December 2008, vol. 2, pp. 610-615. 
[23] T. Zia, "Reputation-based trust management in wireless sensor networks," in Proc. International Conference on Intelligent Sensors, Sensor Networks and Information Processing, December 15-18, 2008, pp. $163-166$.

[24] A. Pirzada, A. Datta, and C. McDonald, "Trust-based routing for ad-hoc wireless networks," in Proc. International Conference on Networks, 2004, vol. 1, pp. 326-330.

[25] X. Li, M. Lyu, and J. Liu, "A trust model based routing protocol for secure ad-hoc networks," in Proc. Aerospace Conference, 6-13 March 2004, vol. 2, pp. 1286-1295.

[26] N. Marchang and R. Datta, "Light-weight trust-based routing protocol for mobile ad-hoc networks," Information Security, vol. 6, no. 2, pp. 77-83, June 2012.

[27] QualNet. (Dec. 6, 2013). Scalable Network Technology. [Online]. Available: http://web.scalable-networks.com/content/qualnet

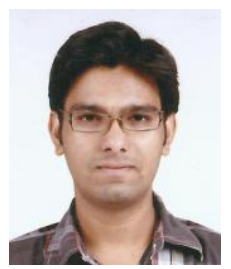

Vinesh H. Patel was born in Bharuch, India, in 1990. He received his B.E. degree in information technology from Veer Narmad South Gujarat University, Surat, India in 2011, and M.Tech. degree in computer engineering from Sardar Vallabhbhai National Institute of Technology, Surat, Gujarat, India in 2014

During 2011-2012, he worked as a software engineer in Emeasurematics, Mumbai. His current research interests include wireless networks, data mining and machine learning.

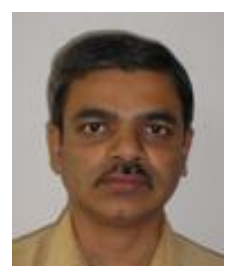

Mukesh A. Zaveri obtained his $\mathrm{PhD}$ degree from Indian Institute of Technology, Bombay in 2005.

$\mathrm{He}$ is serving as an associate professor at Computer Engineering Department, Sardar Vallabhbhai National Institute of Technology, Surat, Gujarat, India from 1993. His research interests are signal processing, wireless network and machine learning.

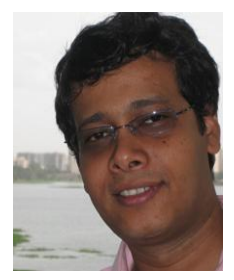

Hemant Kumar Rath is a senior member IEEE, member IARCS. He held MTech and PhD from IIT Bombay, India (both in Communication Engineering) and BE in EL\&TCE from VSSUT Burla (formerly UCE Burla), Sambalpur, Odisha. He has close to 15 years of experience in academics, research and industry. Hemant is a senior scientist at CTO Networks Lab, TCS Bangalore, where he is working since Dec. 2010. His current research interest includes: SDN, wifi offloading, QoS in networks, LTE/WiMAX scheduling, self-optimization, propagation model design, speech processing, M2M communication, cloud computing, modelling of social network traffic etc. He has published many research papers and presented many talks in national and international conferences/seminars such as IEEE Globecom, ICC, PIMRC, COMSWARE, COMSNETS, NCC, ITU-T, BWCI-COAI Workshop etc., and has filled several patents through TCS. He is also participating in national and international standardization activities (IoT - GISFI, IoT - ITU-T, DOSTI, TSDSI-3GPP) in the areas of networking and communication. 